\title{
LA DESLOCALIZACIÓN DE EMPRESAS COMO FENÓMENO DE LA GLOBALIZACIÓN: SUS EFECTOS EN EL CAPITAL INTELECTUAL
}

\author{
Dr. Pedro López Salazar (*) \\ E-mail:pelopez@unex.es \\ Msc. João B. M. Zabot (**) \\ E-mail: jbzabot@unex.es
}

\begin{abstract}
RESUMEN
Globalización es una de las palabras más comentadas hoy en día. También es vista como generadora de muchos problemas empresariales y de solución para tantos otros. La globalización es, en resumen, el resultado del avance tecnológico, de la apertura de las fronteras económicas desde el término de la segunda guerra mundial, de los movimientos internacionales de capital y de la propia liberalización interna en los países, que se llevó a cabo en función de la liberalización externa.

La intensidad de la globalización puede ser observada en cuatro aspectos principales, que son la globalización comercial, financiera, de mano de obra y la globalización tecnológica. En este entorno, en el curso de los últimos años, hemos tenido una significativa ampliación de la diferencia entre los activos de las empresas registrables en el balance de situación y el verdadero valor de mercado. Esta diferencia ha llevado a varios estudiosos a identificar el componente de este valor adicional como un activo intangible, o capital intelectual. Por otro lado, uno de los procesos más recientes en la era de la globalización es la deslocalización de empresas, generalmente multinacionales, que trasladan sus centros de trabajo desde países desarrollados a países con menores costos de mano de obra, provocando en muchos casos un despido masivo de trabajadores. Considerándose que parte sustancial del capital intelectual está reflejado en los conocimientos, habilidades, competencias y valores inherentes a los individuos y grupos que componen una organización, este trabajo intenta -tras definir los procesos de generación del conocimiento organizacional a través del aprendizaje colectivo de las personas o grupos que componen la organización- verificar los modelos de medición de capital intelectual existentes, y analizar las características de la actual deslocalización de empresas, identificando los componentes del capital intelectual afectados en este proceso, en especial los inherentes a las personas.

El principal aporte de este trabajo es, precisamente, la propuesta y descripción de los elementos integrantes del capital intelectual que pueden ser afectados como consecuencia del fenómeno de la deslocalización de empresas, además de apuntar los principales factores involucrados en este proceso, en este nuevo reto de las organizaciones.
\end{abstract}

Palabras Clave: Aprendizaje Organizativo, Capital Intelectual, Deslocalización de Empresas, Globalización.

(") Doctor y Magister en Ciencias Administrativas. Profesor de la Facultad de Ciencias Económicas (Universidad de Extremadura España)

$($ (*) Doctor en Marketing y Comercio Internacional. Magister en Ciencias. Profesor de la Universidad de Extremadura España. 


\begin{abstract}
The globalization phenomena may be described as an increasingly close international integration of markets for goods, services and factors of production, labor and capital, guided by the evolution in information and telecommunications technology. For sure it is not a today's created phenomena, once many of it's characteristics already exist in the land's discovery era, at sixteenth century. The intensity of globalization may be analyzed by commercial and financial relations, workers and labor flows, and technological aspects. It's actuation range goes trough complex processes in technological, economics, politics and cultural dimensions.

In this environment, in the course of the last years there has been a significant increasing in the difference among the assets of the companies registered in their accounting balance, and their true market value. This difference has led several specialists to identify the components of this additional value as intangible assets, or intellectual capital. However, one of the most recent processes in the globalization era is usually the move of companies, generally multinational, that transfer their work centers from developed countries to countries with smaller manpower costs, with a massive discharge of people that were working in them.

Considering that substantial part of the intellectual capital is reflected in the knowledge, abilities, competences and inherent values of the individuals and groups that compose an organization, this work intends, after defining the processes of generation of the organizational knowledge through the collective learning of people or groups that compose the organization, verify existing intellectual capital measurement tools, and after analyzing the characteristics of the current move out of companies, to identify the components of the intellectual capital that get lost in this process, mainly that ones inherent to the persons.
\end{abstract}

The main contribution of this work is, in fact, the proposal and description of those elements of the Intellectual Capital that may be affected as consequence of the phenomenon of companies move out, and the identification of the main factors inherent to this process in this new organizations challenge.

Key Words: Organizing Learning, Intellectual Capital, companies move-out, Globalization.

\section{INTRODUCCION}

El fenómeno globalización es caracterizado como la creciente internacionalización e integración de los mercados de bienes, servicios, trabajo y capital; fundamentalmente amparado por los avances en las tecnologías de información y telecomunicaciones. En verdad no es un fenómeno que haya surgido en nuestros días, sino que algunas de sus características ya se hacían presentes desde la era de los descubrimientos, en el siglo XVI.

La intensidad de la globalización puede ser analizada en términos de relaciones comerciales, financieras, flujo de mano de obra y aspectos tecnológicos. En cuanto a su alcance, envuelve una serie de procesos complejos en las dimensiones tecnológica, económica, política y cultural.

La evolución de las relaciones mundiales en el proceso de globalización pasa por tres períodos distintos: el período anterior a 1914, el período entre las dos guerras mundiales, y el período desde 1944 hasta nuestros días. En el primer período hubo un marco que fue el Trata- do de Libre Comercio entre Francia e Inglaterra; en el segundo se produjo una regresión del comercio mundial y cierre de los mercados de capital, y el tercero se caracterizó inicialmente por la creación de los organismos internacionales como el Fondo Monetario Internacional y el Banco Mundial, y es lo que vivimos hasta hoy.

A pesar de ser un fenómeno antiguo, globalización es un término reciente, de los años 90 , que se puede describir como una creciente integración internacional de los mercados de bienes, servicios y factores de producción, trabajo y capital, integración cada vez más basada en liberalizaciones comerciales, desregulación de los movimientos financieros, facilidad de incorporación de nuevas tecnologías y división internacional del trabajo, y una creciente tendencia a una deslocalización de unidades productivas o de servicios de empresas hacia países en desarrollo con menores costes de mano de obra (Bordo, 2002).

Respecto al proceso de multinacionalización, según Rodríguez (2004), la multinacional es la empresa dominante en la economía glo- 
bal. Considerando multinacional a toda empresa que tiene activos o empleados en más de un país, independientemente de su tamaño (algunas de ellas emplean menos de 250 personas), existen más de 50,000 empresas clasificadas en esta categoría, controlando medio millón de filiales en todo el mundo, aproximadamente. Las 500 multinacionales más importantes controlan casi la mitad de todo el comercio del planeta.

Sin embargo, una de las principales características de la globalización es que cerca de $80 \%$ del comercio mundial está en las manos de multinacionales o de sus filiales, existiendo una tendencia a aumentar las transferencias de actividades de producción de estas empresas hacia otras localidades foráneas buscando, principalmente, menores costos de mano de obra (Aktouf, 2004).

El proceso más reciente procedente de la globalización es la deslocalización, entendiéndose como tal el proceso por el que algunas empresas, generalmente multinacionales, trasladan sus centros de trabajo en países desarrollados a países con menores costos laborales, legislaciones menos estrictas con la protección del medio ambiente, o condiciones de trabajo más flexibles, que permitan menos seguridad en el trabajo y mayor jornada laboral (Aktouf, 2004; Guarasa, 2002; Rodríguez, 2004).

Con respecto a las consecuencias de la deslocalización, cabe mencionar:

- Aumento del paro en el país de origen.

- Creación de empleo precario en el país de destino.

- Contaminación y destrucción del medio ambiente al carecer el país de destino de controles ambientales o de sensibilidad para evaluar los daños apropiadamente, entre otros (Aktouf, 2004).

Es obvio que las empresas se deslocalizan en búsqueda de más beneficios o menores costos, pero ¿cuál es su pérdida en términos del conocimiento y del capital intelectual que poseen las personas que se quedan en su país de origen? ¿cuáles son sus problemas para establecerse en un nuevo entorno cultural? ¿cuáles son las ventajas para los países de destino? ¿cuáles son los retos de las empresas en este proceso?

Considerándose que los conceptos de gestión del conocimiento están intrínsecamente li- gados al concepto de aprendizaje organizativo, y el creciente interés y la introducción de las ideas del capital humano como parte principal de los activos intelectuales importantes en la economía actual (Sveiby, 1998; Stewart, 1999; Nonaka y Takeuchi, 1997), este trabajo tiene como objetivo principal la identificación de los componentes del capital intelectual, principalmente del capital humano, que pueden ser directamente afectados por los procesos de deslocalización de las empresas.

\section{EL CONOCIMIENTO DE LA ORGANIZACION}

La creación del conocimiento puede ser definida como la capacidad de una empresa de crear conocimiento, distribuirlo por toda la organización, e incorporarlo a productos, servicios y sistemas.

Una vez que el conocimiento es creado por los individuos, la creación del conocimiento organizativo debe ser entendida como

“... un proceso que amplía por toda la organización el conocimiento creado por los individuos, transformándolo en parte de su red de conocimientos. Este proceso ocurre dentro de una comunidad de interacción en expansión, que sobrepasa niveles y fronteras interorganizativos" (Nonaka y Takeuchi, 1997, p. 65).

En el modelo propuesto por estos autores -que enfatiza la dimensión epistemológica que representan las categorías básicas de la naturaleza del conocimiento-, la creación del conocimiento se efectúa a través de la interacción social entre el conocimiento tácito (conocimiento personal incorporado a la experiencia individual que integra factores intangibles como creencias personales, perspectivas, sistemas de valores y experiencias individuales), y el conocimiento explícito (aquel que se puede articular en lenguaje formal, afirmaciones gramaticales, especificaciones, expresiones matemáticas, manuales y otros medios).

La interacción entre los dos tipos de conocimiento es continua y dinámica, y es determinada por los cambios entre distintos modos de conversión del conocimiento, guiados por factores externos a la organización, por lo que los autores la llaman espiral de creación del conocimiento (por su continua y dinámica interacción). 


\section{Gráfico 1}

El Sistema de creación del conocimiento en la organización

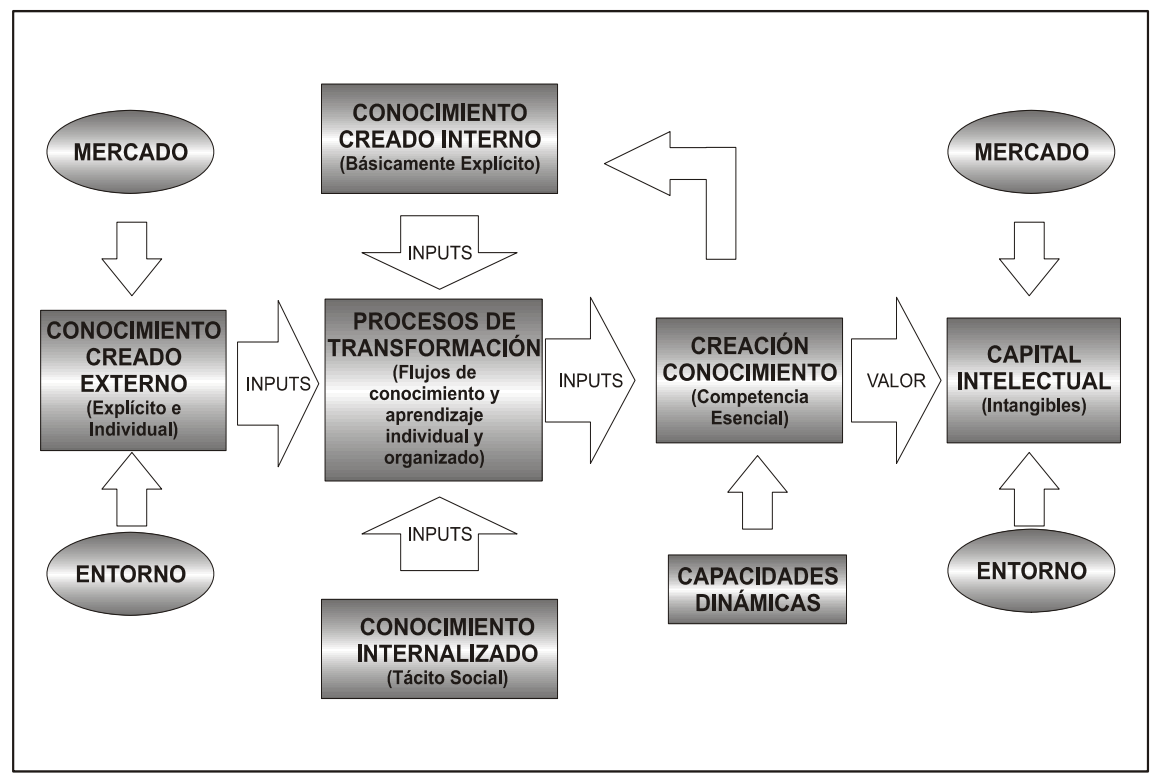

Fuente: Bueno 2002

Siendo que el conocimiento es considerado un conjunto de valores en la empresa, el proceso de su creación suele ser llamado sistema de creación del conocimiento, que puede ser presentado en una visión sistémica, tal como muestra el Gráfico 1.

En este sistema de creación de valor en la empresa, que está basado en el conocimiento, se pueden observar las entradas, que son conocimiento captado en el exterior o dentro de la propia organización; el proceso de transformación de los conocimientos internos y externos; y las salidas, que son el resultado del proceso de creación de nuevos conocimientos, identificados como activos intangibles, parte integrante del concepto de Capital Intelectual.

\section{EL CAPITAL INTELECTUAL}

En la nueva sociedad diversos estudios nos muestran que el gran diferencial competitivo de las empresas y de los países no es ya la mano de obra barata o los recursos naturales, sino su capacidad de generar conocimiento y producir innovación. Las empresas importantes comienzan a ver el proceso de desarrollo de sus recursos humanos como estratégico para su supervivencia y competitividad (Sveiby, 2000; Stewart, 1999).

Los mercados, cada vez más competitivos por fuerza de la globalización y los avances tec- nológicos, han llevado a las organizaciones a una dependencia cada vez mayor de la calidad del conocimiento que se utiliza en sus procesos corporativos y empresariales. El reto de utilizar el conocimiento insertado en la empresa para crear ventajas competitivas se torna cada vez más el punto fundamental para su supervivencia.

Según Sveiby (1998), las estrategias basadas en la información generan ingresos intangibles a partir de los consumidores, y las estrategias basadas en el conocimiento generan una variedad mucho más grande de ingresos intangibles, que vienen de los clientes, de los proveedores y, principalmente, de las propias personas que componen la organización.

De acuerdo con el Comité Europeo de Normalización (CEN) ${ }^{1}$, los valores agregados por el conocimiento al valor de la compañía ocurren en cinco dimensiones:

- Dimensión Financiera. Las inversiones en conocimiento proporcionan resultados directamente en la reducción de costos o incrementos de ingresos.

- Innovación. Cuando se consigue efectivamente crear, compartir y aplicar el conocimiento; de hecho las organizaciones consiguen desarrollar y lanzar nuevos productos y servicios más rápidamente.

- Procesos. El conocimiento normalmente es o debería ser la parte principal de la mayoría 
de los procesos, en cuanto al desarrollo de productos, marketing y ventas, los servicios ofrecidos a los clientes y la conquista de nuevos clientes, convirtiéndose en más eficientes y eficaces.

- Clientes. El conocimiento ayuda a crear el capital de clientes, a través de un mejor conocimiento de éstos y de sus necesidades, permitiendo perfeccionar los productos y los servicios ofrecidos.

- Personas (empleados). Muchos empleados pueden ser considerados trabajadores del conocimiento, siendo una efectiva gestión de este conocimiento el medio eficaz para que ellos puedan desarrollar y utilizar sus talentos hacia la creación del conocimiento organizacional.

Estas cinco dimensiones están directamente relacionadas con el Capital Intelectual de la empresa, que incluye el propio conocimiento individual de los empleados, datos e información de procesos, especialistas, productos, clientes y competidores, y propiedades intelectuales como las patentes y licencias. El Capital Intelectual engloba, por tanto, comprensiones, percepciones y tecnologías. Éste es el componente que define la diferencia entre el valor de la contabilidad y el valor de mercado de una compañía.

En su guía, el CEN subdivide el Capital Intelectual en tres áreas diferentes:

- Capital Humano. Está formado por las habilidades, el talento, el conocimiento y la especialización del empleado. Puede definirse como la capacidad colectiva de la organización para extraer de la base de conocimiento de sus individuos las mejores soluciones para el cliente.

- Capital Estructural. Puede llamarse así al conocimiento que ha sido adquirido e institucionalizado dentro de la estructura, procesos y cultura de una organización. Incluye las patentes, los copyrights, el software en propiedad, las marcas, secretos comerciales y el know-how general de la organización. Esto puede almacenarse en forma de procedimientos documentados, base de datos, sistemas expertos, softwares de apoyo a las decisiones como los sistemas de Gestión del Conocimiento. Además de esto, el Capital Estructural va construyéndose por medio de las relaciones con los proveedores, el gobierno y los inversores.

- Capital de Clientes. Se refiere a una red de clientes satisfechos y leales a la organización. El valor del Capital Intelectual de la organización debería medirse por la cantidad y calidad de las relaciones con clientes que se han conseguido con el paso de los años. Lo que tiene valor es la confianza del cliente en los productos y servicios prestados.

Pero, ¿cómo se mide el Capital Intelectual de una organización? Existen varios modelos de medición del Capital Intelectual de las empresas, algunos de ellos con la finalidad principal de medir directamente los activos intangibles de las organizaciones, y otros desarrollados como instrumentos de dirección estratégica de la empresa, contemplando de alguna forma las dimensiones intangibles o la creación de valor basada en conocimientos.

A partir de un amplio estudio de los modelos existentes, el Centro de Investigación sobre la Sociedad del Conocimiento (CIC $)^{2}$ desarrolló el Modelo Intellectus ${ }^{3}$, en base a un modelo inicial (Intelect), que reafirma la participación fundamental del capital humano en la formación del capital intelectual de las empresas.

El modelo Intelect original, presuponiendo que el capital intelectual es el conjunto de activos de una empresa que, aunque no se refleja en los archivos de contabilidad tradicionales, generan o generarán valor para la compañía en el futuro, agrupaba el capital en tres bloques: el capital humano, el capital estructural y el capital relacional.

A partir de esta triple naturaleza de los activos intelectuales, el Modelo Intellectus continúa basado en los tres bloques previamente mencionados, pero descompone el capital estructural en capital organizativo y capital tecnológico, subdividiendo el capital relacional en capital de negocio y capital social.

Dentro del Modelo Intellectus, el componente Capital Humano se refiere al conocimiento (explícito, tácito, individual o social) que tienen las personas o los grupos de personas, así como su capacidad de generar este tipo de conocimiento dentro de lo que pueden ser considerados los siguientes elementos: 
- Valores y Actitudes (Ser+Estar). Identificación y compromiso con la organización, automotivación (impulsos, deseos, aspiraciones), satisfacción (grado de vinculación y participación en las tareas), sociabilidad (facilidad de trato y relación con las personas), flexibilidad y adaptabilidad (actitud positiva ante el cambio), y creatividad (proceso por el que se facilita la aparición de nuevas ideas).

- Aptitudes (Saber). Educación reglada (conjunto de conocimientos explícitos derivados de procesos reglados que poseen las personas), formación especializada (conocimientos específicos de un área concreta), experiencia (saber que se adquiere con la práctica), y desarrollo personal (conocimientos derivados de procesos informales de relación con el entorno).

- Capacidades (Saber hacer). Aprendizaje (capacidad de responder positivamente al cambio y adquirir nuevas competencias y conocimientos), colaboración (capacidad de desempeñar trabajos en equipo y tomar decisiones en grupo), comunicación (capacidad de intercambiar conocimiento) y liderazgo (habilidad de influenciar positivamente en los demás).

El Capital Organizativo es el conjunto de intangibles que estructuran y desarrollan la actividad de la organización, y se compone de los elementos:

- Cultura. Homogeneidad cultural, evolución de valores culturales, clima social, filosofía de negocio.

- Estructura. Diseño y desarrollo organizativo.
- Aprendizaje organizativo. Capacidad de la organización de adquirir nuevas competencias y conocimientos a través de los entornos de aprendizaje, rutinas y procedimientos organizativos, procesos de creación, captación, desarrollo y transmisión del conocimiento.

- Procesos. Conjunto de actividades que configuran las operaciones organizativas dirigidas al cliente interno (los miembros de la organización), al cliente externo (los consumidores de sus productos), y a los proveedores (participación de los proveedores en los procesos productivos de la organización).

El Capital Tecnológico se refiere a los intangibles directamente relacionados con el sistema técnico de operaciones de la empresa, como son:

- Esfuerzos en investigación (aumento del volumen de conocimientos técnicos).

- Desarrollo. Incorporación de estos conocimientos en nuevas aplicaciones y nuevos productos o servicios.

- Innovación. Lanzamiento de bienes y servicios tecnológicamente nuevos, así como los esfuerzos destinados a compras de nuevas tecnologías, el volumen de conocimientos protegidos legalmente en la forma de propiedad intelectual e industrial (patentes y modelos, marcas registradas, licencias, y dominios en Internet), y los propios resultados de la innovación.

Respecto al Capital Negocio y Capital Social, la referencia está en las relaciones con clien-

Cuadro 1

Síntesis de los modelos de medición del Capital Intelectual

\begin{tabular}{|c|c|c|c|c|c|c|c|c|c|c|}
\hline Componente & Bontis & Skandia & $\begin{array}{l}\text { Tech. } \\
\text { Broker }\end{array}$ & $\begin{array}{l}\text { Univ } \\
\text { Ontario }\end{array}$ & $\begin{array}{c}\text { Canadian } \\
\text { Bank }\end{array}$ & Sveiby & Nova & CEN & Intellect & Intellectus \\
\hline Humano & 0 & 0 & 0 & 0 & 0 & 0 & 0 & 0 & 0 & 0 \\
\hline Mercado/Cliente & 0 & 0 & 0 & 0 & 0 & 0 & 0 & 0 & 0 & \\
\hline Financiero & & 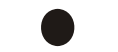 & & & & & & 0 & & $\mathrm{O}$ \\
\hline Proceso & 0 & 0 & & & & & & 0 & & 0 \\
\hline $1+D$ & & 0 & & & & & & 0 & & 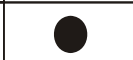 \\
\hline Propiedad Intelectual & & & 0 & & & & & & & \\
\hline Infraestructura & 0 & & 0 & 0 & 0 & 0 & 0 & & 0 & \\
\hline Desempleo & & & & 0 & 0 & & & 0 & & \\
\hline
\end{tabular}

Fuente: Elaboración propia a partir de los componentes de los diferentes modelos analizados. 
tes (base de clientes, lealtad, satisfacción, procesos de relaciones y red de distribución); relaciones con proveedores (grado de formalización de la relación, capacidad de respuesta); relaciones con accionistas, instituciones e inversores; relaciones con competidores; relaciones con instituciones de mejora de la calidad, con la administración pública, con medios de comunicación e imagen corporativa y con los órganos de defensa del medio ambiente.

A modo de síntesis, los componentes de los principales modelos de medición de capital intelectual pueden verse en el Cuadro 1.

Se puede notar, en todos los modelos, la presencia fundamental del capital humano (además del capital de clientes) como componente de los activos intangibles y, consecuentemente, del capital intelectual de las empresas. El capital humano es, por tanto, una de las bases principales para la formación del Conocimiento Organizativo, dentro del proceso identificado por Hofstede (1991) como aprendizaje, o en otros términos, de todo un proceso generado por las personas de una organización a través del cual se crean una serie de valores que pasan a pertenecer a la propia organización, así como otros valores inherentes a las propias personas, grupos o equipos y que sólo a ellos pertenece, porque muchas veces es imposible explicitarlos.

\section{EL CAPITAL INTELECTUAL Y EL APRENDIZAJE ORGANIZATIVO}

El concepto de aprendizaje como proceso de formación cultural es presentado por
Hofstede (1991) al describir la cultura como una programación mental que no es innata del ser humano, sino obtenida a través del aprendizaje de una serie de informaciones, símbolos, valores y prácticas individuales y de los grupos y equipos que son agregados a las propias características heredadas por las personas formando su personalidad.

Considerando que las organizaciones están compuestas por personas, a su vez reunidas en grupos, equipos o departamentos, y que estas personas no sólo son la base del funcionamiento y de la existencia de las organizaciones, sino que son los poseedores de la gran fuente de conocimiento tácito en ellas existente, nosotros podríamos extrapolar este concepto para el nivel organizativo, en donde el conocimiento organizativo puede interpretarse como resultado de un proceso cultural de aprendizaje entre las personas, equipos o departamentos, proceso que forma -en último análisis- el conocimiento y una parte considerable del Capital Intelectual de la compañía (Ver Gráfico 2).

En este sentido, Prusak (1996) afirma que el conocimiento y la especialización son creados y residen en las mentes de las personas, y a ellas están, en cierta forma, limitados. Según Sveiby (1998), la especialización es un conocimiento más profundo en ciertos campos de actividades, que proporciona nuevos conocimientos y soluciones basados en experiencias, entrenamientos y educación continua, y que pertenece y permanece con las personas, quienes se lo llevan con ellas cuando deciden marcharse de la empresa.

Gráfico 2

Aprendizaje Organizativo

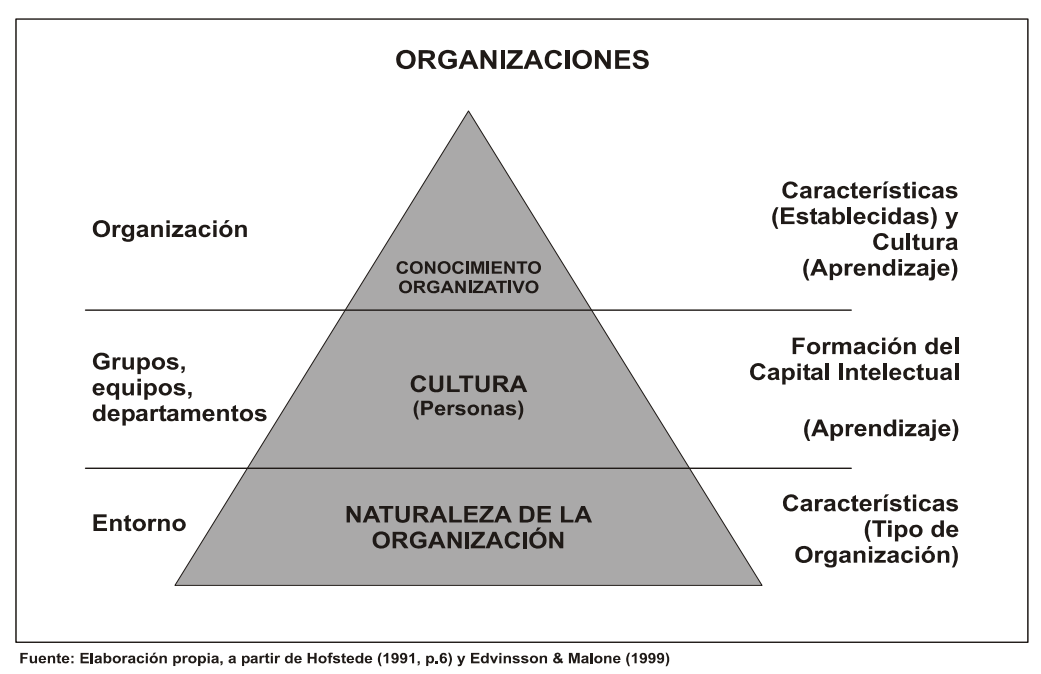


Considérase el proceso de aprendizaje organizativo como el proceso dinámico de interacción entre individuos, grupos y la propia organización, cada uno de ellos trasmitiendo y compartiendo informaciones y conocimientos con todos los otros en los dos sentidos: desde los individuos hasta la organización, pasando por los grupos (feed-forward), y en el sentido contrario, desde la organización hasta los individuos (feedback), conforme se presenta en el Gráfico 3.

\section{Gráfico 3}

Esquema del Aprendizaje Organizativo

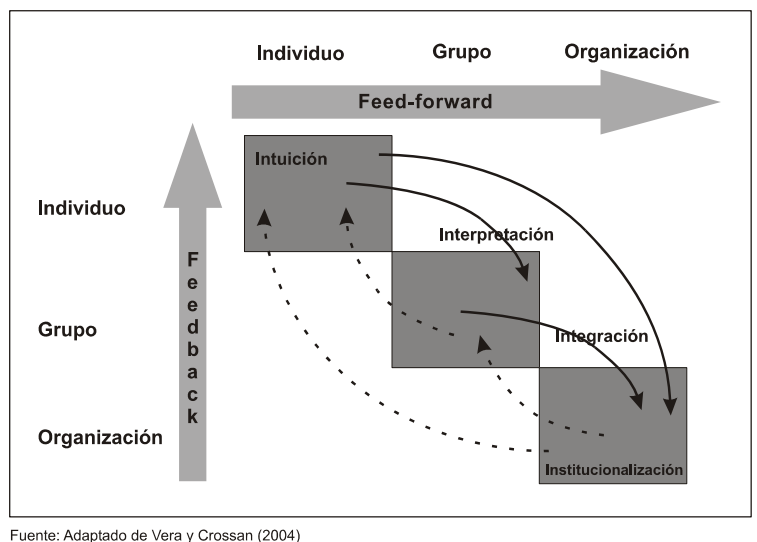

Como demuestra el esquema, el aprendizaje organizativo es un proceso dinámico que no ocurre tan solo una vez, sino que es continuo en el tiempo, a través de todos los niveles de la organización. La dinámica se crea por el flujo en los dos sentidos, de los individuos hasta la organización por los procesos llamados feed-forward (alimentación hacia adelante) y feedback (alimentación hacia atrás, o retroalimentación). La generación del conocimiento en este proceso ocurre por medio de la intuición, interpretación, integración e institucionalización de los conocimientos individuales, grupales y organizativos.

El proceso de aprendizaje organizativo, por tanto, es la base para la formación del capital intelectual de las empresas, sea del propio capital humano, del capital estructural o del capital relacional. Según Miller y Wurzburg (en Edvinsson y Malone, 1999), es un proceso de generación de conocimiento y de capital intelectual a partir de los miembros de la empresa, de lo que dependen cada vez más las organizaciones para su supervivencia económica, cuya suma incluye una parcela considerable de conocimientos que permanecen detenidos en las pro- pias personas y no en la organización. Esta parcela de conocimientos suele, a su vez, ser llevada por los trabajadores cuando salen de la empresa, independientemente del motivo por el que se marchan.

\section{LA DESLOCALIZACIÓN DE LAS EMPRESAS}

Deslocalización, de acuerdo con el análisis morfológico de la palabra, es fijar algo fuera de unos límites determinados. Esto, aplicado al mundo empresarial, significa fijar las empresas fuera de los límites de un determinado país, o, dicho de otro modo, mover una unidad productiva de una empresa de un país a otro.

Con respecto al proceso de deslocalización, una definición amplia del concepto es dada por Guarasa (2002), quien considera la deslocalización de la inversión directa extranjera como el proceso por el cual una empresa de capital foráneo, situada en un país, toma la decisión de abandonar su actividad parcial o totalmente para situarla en otro país, cualquiera que sea la causa que la determine.

Según Guarasa $(2002,28)$, se puede entender deslocalización empresarial como

“... el traslado de actividades productivas desde países industrializados a países en desarrollo caracterizados por poseer niveles sensiblemente inferiores en su renta per cápita $y$, por consiguiente, en el salario medio de la población empleada."

En los 90 -años de mayor expansión económica en los países desarrollados, de impulso de la integración económica internacional y de avance en las libertades políticas y en la consolidación institucional de muchos países en desarrollo- el proceso de deslocalización de las actividades empresariales ha adquirido un gran vigor. La reducción de las barreras comerciales, en especial de los aranceles, ha facilitado la entrada en los diversos mercados nacionales. La mayor presión competitiva que crea un mercado más global ha llevado a la reducción de los costes de las empresas, entre ellos los laborales, y ha aumentado el atractivo de fabricar en zonas con salarios más bajos. Además, la emergencia de nuevos mercados con claras perspectivas de expansión (China, India y los países de Europa central y oriental) ofrecen grandes oportunidades de negocio (Fernández y Myro, 1996). 
Este fenómeno ocurre, sobre todo, en los sectores tecnológicamente más avanzados, siendo algunos de los casos más conocidos en España los de Alcatel, Ericsson, Lucent Technologies, Delphi, Lear, Valeo, Samsung, Phillips o Siemens.

La experiencia sobre el caso español se refleja en un estudio realizado por Fernández y Myro (1996) a mitad de los 90, tras la preocupación del Comité de las Regiones de la Unión Europea sobre la posibilidad de que las diferencias de regulación laboral y social entre países miembros favorecieran las deslocalizaciones. En este estudio, se verificó que entre 1990 y 1995 se produjeron 174 casos de deslocalización, cien de ellos en España. Del total, 19 supusieron el cierre de las instalaciones; otros 19 pasaron a manos de propietarios nacionales. En los demás, se cerraron líneas de productos o instalaciones específicas. De los casos correspondientes a España, el $75 \%$ eran firmas extranjeras y el resto pertenecían a empresas españolas y se localizaban en los sectores más tradicionales, como alimentos, calzado y textil, fundamentalmente.

Según Ceballos Fernández y Colmena (2004), la deslocalización se basa fundamentalmente en los costes laborales ${ }^{4}$. Así Intel invertirá 200 millones de dólares en India para fortalecer su centro de diseño de software y triplicará su número de ingenieros a tres mil; Microsoft prevé doblar el número de empleados en India; a finales del 2007 el $23 \%$ de trabajadores del sector estadounidense de TI estarán en países con sueldos más bajos, respecto al 5\% actual; en los empleos de los "Call Centers" de España, estos se están deslocalizando a países de Latinoamérica y a Marruecos, por el conocimiento del idioma - por lo que cobra un teleoperador en España se pueden pagar tres en Argentina, cinco en Marruecos y casi siete en Colombia o Perú.

Existe, según diferentes autores (ex. Aktouf, 2004; Guarasa, 2002), una pérdida de empleo en todos los países del mundo, habiéndose provocado en los últimos siete años una reducción de empleo de un $11 \%$ en todas las regiones del planeta, y el reconocimiento por parte de la Organización Internacional del Trabajo (OIT) de la pérdida global de empleo en 185,9 millones de personas, además de que la pérdida de empleo también afecta a países como Hungría o Checoslovaquia, en teoría beneficiarias de la deslocalización.
En América, una comprobación de estos hechos es que en cuanto el Tratado de Libre Comercio de América del Norte - NAFTA (acuerdo entre Estados Unidos, Canadá y México) estuvo ratificado, comenzó el éxodo de plantas completas de Estados Unidos -sobre todo de aquellas que usualmente procedían a terminar los productos- hacia territorio mexicano, donde leyes especiales las protegían, eximían de impuestos y negaban a los asalariados muchos derechos, sobre todo el de sindicalizarse. Según Aktouf (2004), la atracción ejercida por los bajos salarios mexicanos, las renuncias fiscales y la eliminación de derechos de aduana ha llevado a una transferencia masiva de la industria de Estados Unidos.

"En seis años, se cerraron 2,300 fabricas en los Estados Unidos, o se deslocalizaron, eliminando más de 230,000 empleos en este país". (Aktouf, 2004, 91).

En términos de despido masivo de personas, según el autor, en los últimos diez años las 500 mayores organizaciones de los Estados Unidos despidieron un promedio de 400,000 empleados al año.

Las grandes corporaciones de Estados Unidos miran cada vez con más interés hacia el otro lado del Pacífico para concentrar toda su producción en los países del Sudeste Asiático, donde la mano de obra es mucho más barata y pueden conseguir ahorros de hasta el $80 \%$, y también hacia América Latina.

Con el fin del Acuerdo Multifibras ${ }^{5}$ que restringía las exportaciones de productos textiles de los países en desarrollo en enero de 2005, los sindicatos prevén que 30 millones de empleos del sector textil migrarán a China, y a la vez con perjuicio inclusive a países en desarrollo de Asia, Centroamérica y África. Este hecho es también confirmado por recientes noticias que afirman que, a pesar del acuerdo comercial $\mathrm{CAFTA}^{6}$, América Central perderá su cuota de mercado de cualquier forma, en función de las extremas ventajas de los costos laborales en China. Es interesante observar que hasta las propias empresas chinas se están deslocalizando, pues recientemente algunas empresas chinas que cultivaban verduras en España para venderlas a restaurantes chinos de Europa, han decidido trasladar la producción a Turquía. 
Indican los resultados de una investigación efectuada en 168 fábricas de 140 empresas que se deslocalizaron para otros países, dentro y fuera de la Unión Europea, la mayoría multinacionales del sector de alta o media tecnología, las razones declaradas para abandonar o mover las actividades de producción (Fernández y Myro, 1996). En este trabajo, se analizaron, entre otras variables, los factores que determinan la migración (Ver Cuadro 2).

Los resultados muestran que la mayoría de las deslocalizaciones tuvieron dos motivaciones: costos laborales y reorganización estructural. Sin embargo, los costos laborales reflejaron más del
$50 \%$ de la motivación mientras que la reorganización cuenta con un tercio de las respuestas, siendo los otros motivos, a modo individual, prácticamente insignificantes, además de que el término reorganización es muy amplio y puede contener también costos laborales.

Las empresas, por lo tanto, suelen trasladarse a otros países con otras culturas y otros entornos en búsqueda de menores costos, dejando en su país de origen a personas que poseen conocimiento, personalidad y competencias adquiridas con el aprendizaje obtenido durante el tiempo que han permanecido trabajando en ellas.

Cuadro 2

Razones declaradas para abandonar o mover las actividades de producción

\begin{tabular}{|c|c|c|c|c|c|c|c|c|}
\hline \multirow{3}{*}{ Razón declarada } & \multicolumn{8}{|c|}{ Nivel Tecnológico } \\
\hline & \multicolumn{2}{|c|}{ Alto } & \multicolumn{2}{|c|}{ Medio } & \multicolumn{2}{|c|}{ Bajo } & \multicolumn{2}{|c|}{ Total } \\
\hline & Num & $\%$ & Num & $\%$ & Num & $\%$ & Num & $\%$ \\
\hline Reorganización & 32 & 56,1 & 15 & 50,0 & 10 & 12,3 & 57 & 33,9 \\
\hline Costes de producción & 17 & 29,8 & 4 & 13,3 & 64 & 79,0 & 85 & 50,6 \\
\hline Mano de Obra & 15 & - & 4 & - & 61 & - & 80 & - \\
\hline Otros & 2 & - & - & - & 3 & - & 5 & - \\
\hline Resultados económicos negativos & 2 & 3,5 & 4 & 13,3 & 0 & - & 6 & 3,6 \\
\hline Obsolescencia de las plantas & 2 & 3,5 & 1 & 3,3 & 1 & 1,2 & 4 & 2,4 \\
\hline Proximidad a mayores mercados & 1 & 1,7 & 1 & - & 2 & 2,4 & 3 & 1,8 \\
\hline Absorción de marcas/plantas/mercado de competidores & - & - & 2 & 6,7 & 1 & 1,2 & 3 & 1,8 \\
\hline Regulamentaciones institucionales & 1 & 1,7 & - & - & 2 & 2,4 & 3 & 1,8 \\
\hline Aspectos tecnoestruturales & 1 & 1,7 & 1 & 3,3 & 0 & - & 2 & 1,2 \\
\hline Absorción de producción subcontratada & - & - & 1 & 3,3 & 0 & - & 1 & 0,6 \\
\hline Menores requisitos ambientales & - & - & 1 & 3,3 & 0 & - & 1 & 0,6 \\
\hline Sin datos & 1 & 1,7 & 1 & 3,3 & 1 & 1,2 & 3 & 1,8 \\
\hline Total & 57 & 100,0 & 30 & 100,0 & 81 & 100,0 & 168 & 100,0 \\
\hline
\end{tabular}

Fuente: Fernández y Myre (1996)

\section{LA PERDIDA DE CONOCIMIENTO EN LOS PROCESOS DE DESLOCALICACION}

$\mathrm{Si}$ bien los conceptos ligados al desaprendizaje suelen llevar al sentido de "olvidar" viejos conocimientos para tomar nuevas decisiones, podemos establecer que, en términos organizativos, el proceso de desaprendizaje puede ser considerado como lo contrario del proceso de aprendizaje organizativo, o sea, la pérdida del aprendizaje colectivo de parte sustancial del capital intelectual. Seria la pérdida de logros pasados, de resultados presentes y futuros del proceso de aprendizaje, así como de los valores creados en las propias personas (la especialización), que se van con ellas cuando salen de la empresa (Bender y Fish, 2000).
Sin embargo, puesto que este conocimiento es el componente fundamental de las variables que componen el capital intelectual de una organización, hay que caracterizarlo en los varios elementos constituidos por estas variables. En esto sentido, una primera aproximación a esta caracterización es presentada por Badaracco Jr. (1992), en la cual distingue dos tipos de conocimiento: conocimiento migratorio y conocimiento insertado, los cuales, en cierta forma, reflejan los conceptos de conocimiento explícito y tácito de Nonaka y Takeuchi (1995). (Ver Gráfico 4).

El conocimiento migratorio es el conocimiento que es descifrable, y se encuentra en paquetes ordenados y móviles como archivos de ordenador, libros y fórmulas, diseños, piezas de 
maquinarías, e incluso algunos que residen en la mente de las personas. Es también el conocimiento contenido en las máquinas, desde las más simples hasta las más sofisticadas, como ordenadores con sistemas expertos. Estos son llamados conocimientos migratorios, porque pueden emigrar con rapidez con la organización.

Gráfico 4

Tipos de Conocimiento

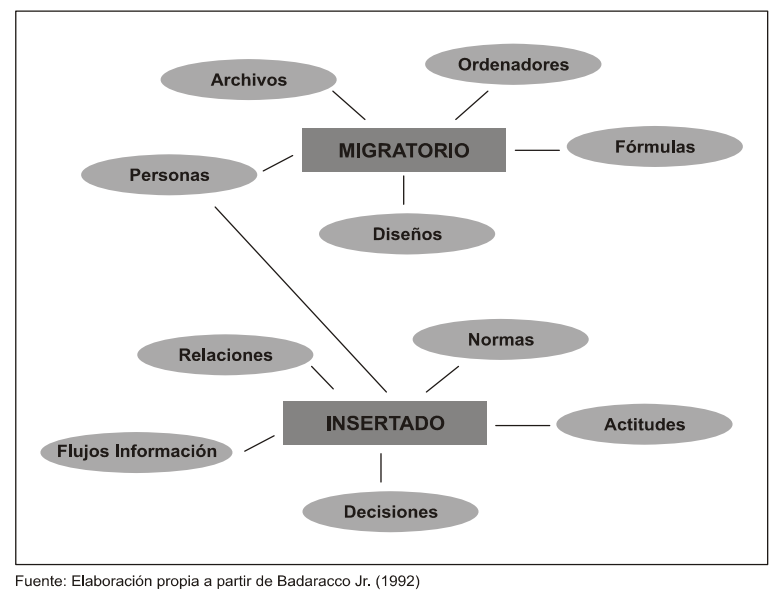

El conocimiento insertado, o conocimiento no migratorio,

“... reside fundamentalmente en las relaciones especializadas entre individuos y grupos $y$ en las particulares normas, actitudes, flujos de información y formas de tomar decisiones que caracterizan los contactos entre ellos." (Badaracco Jr, 1992, 53).

Al contrario que el conocimiento migratorio, es un tipo de conocimiento que se mueve con lentitud. Es un conocimiento ostentado por las personas, vinculado a circunstancias particulares de tiempo y lugar. Según el economista Frederick Hayek (en Badaracco Jr, 1992, 56),

"Para poder valorar adecuadamente este conocimiento sólo necesitamos recordar lo mucho que tenemos que aprender sobre cualquier ocupación después de haber terminado la formación teórica, la gran parte que dedicamos de nuestra vida laboral a aprender determinados trabajos $y$ lo valioso que es en todas las profesiones el conocimiento de la gente, de las condiciones locales y de las circunstancias especiales".

Lo que ocurre en los procesos normales de deslocalización de las empresas es que, tras un despido masivo de personas, existe una consecuente pérdida de los conocimientos inserta- dos y de la cultura inherente a las personas y grupos, componentes directos del capital intelectual en sus distintos elementos adquiridos a través del proceso de aprendizaje (Gráfico 5).

Gráfico 5

Pérdida de conocimientos insertados

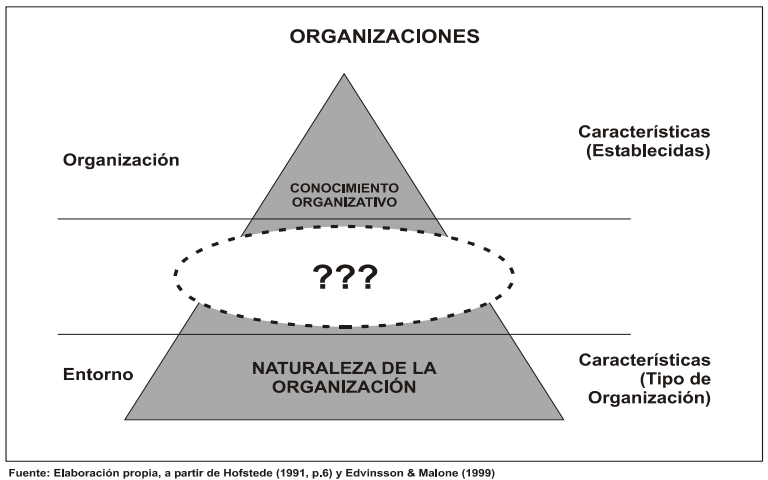

Sin embargo en las empresas en proceso de deslocalización, al trasladarse para otros países y establecerse en nuevos entornos y nuevas culturas, se genera un cambio de personas y de grupos, y un reinicio del proceso de aprendizaje por el mismo sistema continuo de interacción entre las personas y grupos que la componen (Gráfico 6).

Gráfico 6

El nuevo proceso de Aprendizaje

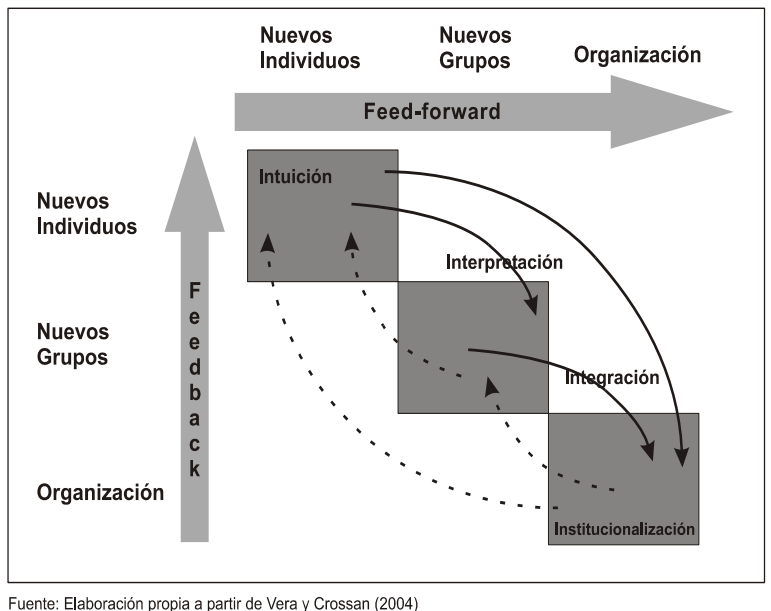

Por ser un proceso inherente al proceso general de aprendizaje organizativo, con la transformación de la empresa en nuevos individuos y nuevos grupos (en su nueva localización), se inicia un nuevo proceso de feed-forward y feedback de las intuiciones, interpretaciones, integraciones e institucionalizaciones de conocimientos. 
ELEMENTOS DEL CAPITAL INTELECTUAL

\section{AFECTADOS EN EL PROCESO DE} DESLOCALIZACION

En los apartados anteriores, a partir del concepto de globalización, hemos hablado del sistema de creación de conocimiento en las organizaciones a través de la creación de valor basada en el aprendizaje organizativo, así como de la generación y elementos que integran el Capital Intelectual. Tras el análisis de distintos modelos existentes de medición del Capital Intelectual, vamos a desagregar los diversos elementos que suelen perderse o pueden ser afectados en el proceso de desaprendizaje organizativo originado por la deslocalización, representados en el Gráfico 7.

Gráfico 7

Elementos del Capital Intelectual afectados por la deslocalización

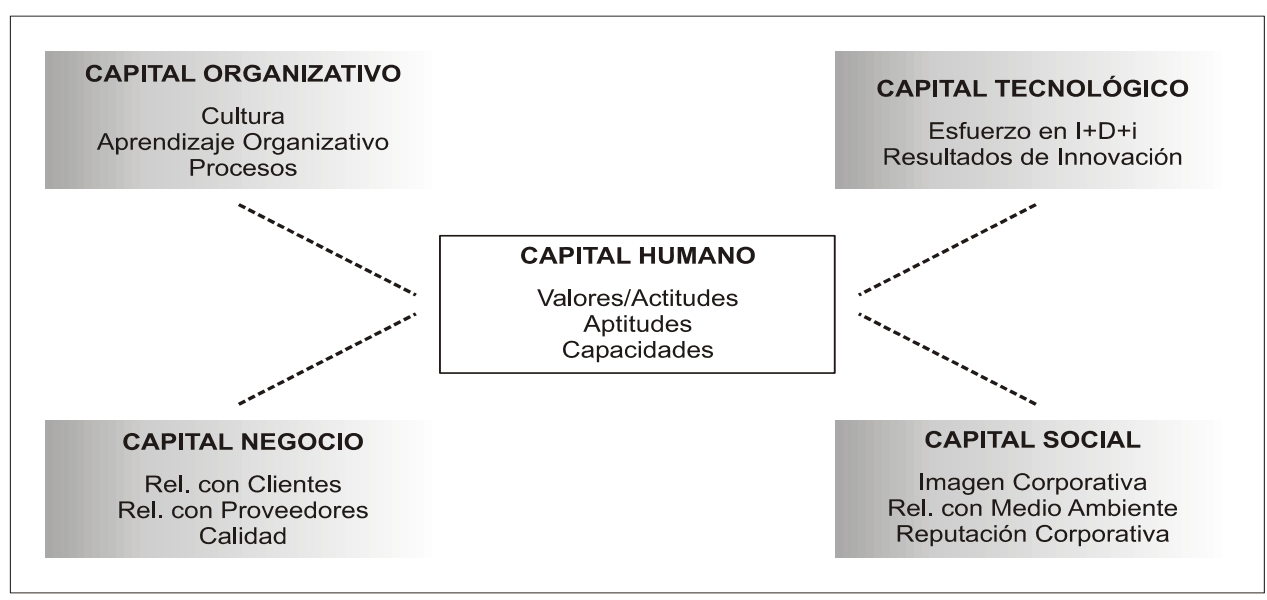

Fuente: Elaboración propia, a partir de Hofstede (1991, p. 6) y Edvisson \& Malone (1999).

Respecto al Capital Humano, que es el conocimiento que poseen las personas y grupos así como su capacidad para generarlos, que se integra en lo que las personas y grupos saben y por la capacidad de aprender y compartir conocimientos, tiene efectos que pueden estar en:

- Valores y Actitudes. Representan los hechos o circunstancias de identificarse y sentirse miembro de la organización; las fuerzas que hacen que las personas desempeñen mejor sus tareas; actitudes positivas, como el sentimiento de pertenencia y compromiso con la organización; automotivación, satisfacción, sociabilidad, adaptabilidad y creatividad.

- Aptitudes. Que hacen referencia al conocimiento que la persona tiene sobre las materias como educación, formación especializada, experiencia y desarrollo personal.

- Capacidades. Refiriéndose al tipo de conocimiento relacionado con la forma de hacer las cosas como competencias, conocimientos, colaboración, comunicación y liderazgo.

También en el Capital Estructural, el conjunto de conocimientos y de activos intangibles derivados de los procesos de acción que son pro- piedad de la organización, existen algunos componentes que suelen ser inherentes a las personas:

a)En el Capital Organizativo. Conjunto de intangibles que estructuran y desarrollan la actividad de la organización:

- Cultura: grado de coherencia, aceptación y compromiso, principios y clima social, visión del negocio, modos y procesos de organización formal de la empresa.

- Aprendizaje organizativo: facilitación de entornos de aprendizaje, captación, transmisión, creación y desarrollo de conocimientos.

- Procesos: participación en los procesos dirigidos hacia clientes internos, clientes externos y proveedores.

b)En el Capital Tecnológico. Intangibles vinculados con el sistema técnico de operaciones de la organización:

- Esfuerzo en I+D+i: personal involucrado en actividades de investigación, desarrollo e innovación.

- Resultados de la innovación: motivación de la plantilla. 
Respecto al Capital Relacional, conjunto de conocimientos que se incorporan a la organización y a las personas como consecuencia del valor de las relaciones con el mercado y con la sociedad en general:

\section{a)En el Capital Negocio.}

- Relaciones con clientes: participación de las personas en los procesos de relacionamiento con los clientes.

- Relaciones con proveedores: participación de las personas en los procesos de relacionamiento con los proveedores.

- Calidad: personas involucradas en los procesos y sistemas de calidad.

\section{b)En el Capital Social.}

- Imagen corporativa: reputación y nombre de la empresa.

- Relaciones con la defensa del medio ambiente: participación de las personas en proyectos de preservación ambiental.

- Reputación corporativa: relaciones que la empresa mantiene con los diferentes agentes sociales (mercados, instituciones, ciudadanos y consumidores).

Todos estos elementos pueden ser afectados directamente, en mayor o menor grado, en los procesos de deslocalización de las empresas cuando cierran sus actividades en un país para establecerse en otro.

\section{CONCLUSIONES}

El conocimiento adquirido a través del proceso continuo de aprendizaje adopta formas tan diferentes que todavía no hay una unidad común de medida de la cantidad de conocimiento existente en un momento dado. También es difícil cuantificar la parte del capital intelectual que se escapa a través de las fronteras en los procesos de deslocalización. Sin embargo, se puede concluir que, dadas las características de los elementos que lo componen -pertenecer en mayor o menor grado a las personas- en todos puede haber una variación en mayor o menor grado.

A pesar de la comprobación de la importancia de las personas como verdaderos agentes y fuente de los activos intangibles de las empresas, clasificando su competencia y sus co- nocimientos como el principal activo invisible (Sveiby, 1998; Drucker, 1999; Stewart, 1999), en los 90 el proceso de deslocalización de las actividades empresariales adquirió gran vigor, sobretodo en sectores tecnológicamente más avanzados, donde inclusive se supone un mayor nivel de conocimientos especializados. Con respecto a este proceso, la decisión de algunas empresas de abandonar su actividad parcial o totalmente en un país para situarla en otro, generalmente está relacionada principalmente a una búsqueda de menores costos laborales, con un consecuente despido masivo de personas en su país de origen, que allí se quedan con sus conocimientos, personalidades y competencias adquiridas en todo un proceso de aprendizaje organizativo individual, en equipos y en la organización como un todo. Lo que ocurre, por tanto, en dichos procesos, es la eliminación de los aspectos individuales y grupales del proceso de aprendizaje organizativo, con una consecuente pérdida de los componentes directos del capital intelectual.

Además de esta evaluación, se puede también verificar que existen algunas paradojas en los conceptos y actitudes de las organizaciones hoy en día pues -a pesar de los conceptos de motivación, valores, necesidad de sinergia empresarial, dedicación de tiempo para aprender y, principalmente, valoración e importancia de las personas y del capital humano- las empresas suelen estar siempre frente a retos como necesidades de reducción de la plantilla, reducción de la estructura y trabajo con menos recursos y menos costos, llevándolas a efectuar el traslado de sus actividades productivas o parte de ellas, a países con menores costos laborales con una consecuente pérdida de parte de su conocimiento organizativo.

Frente al proceso de deslocalización existen preocupaciones a corto plazo que hay que abordar, una vez que todos los cambios que se producen en las ventajas comparativas normalmente poseen costos de ajuste económico. Es posible que un cierto número de puestos de trabajo ya no se necesiten en empresas que proceden, por ejemplo, a deslocalizar servicios, pero probablemente habrá un penoso período de transición mientras la mayoría de los trabajadores afectados por la deslocalización buscan un nuevo empleo. Muchos tendrán quizás que adquirir nuevos conocimientos o trasladarse a nue- 
vos lugares para poder encontrar un nuevo empleo.

A los países destinatarios de las actividades de la producción deslocalizada les pasa lo contrario, pues se crean nuevos puestos de trabajo y se incrementa la producción, como el caso de China con la industria textil, donde se calcula que 20,000 nuevos proveedores chinos concurrirán a partir del termino del acuerdo multifibras para sumarse a los 5,000 actuales, lo que permitirá al país incrementar sus exportaciones entre un 250\% y un 330\% en la próxima década. Según el Banco Mundial, ya en 2010 China pasará a controlar la mitad del mercado mundial textil.

Este es un fenómeno inevitable e irreversible que, en verdad, configura un nuevo marco de competencia para el que las empresas responsables deben prepararse a fondo, a través de la generación de valor añadido a su capital humano mediante la inversión en investigación, desarrollo, innovación tecnológica y formación continua para, de esta forma, contar con una cadena de valor más desarrollada que les permita, a través de producciones de alto valor agregado en diseño y alta calidad, no tener que competir de igual a igual con los bienes provenientes de estos nuevos mercados productivos.

\section{NOTAS}

1 European Committee for Standardization - formado por representantes de Austria, Bélgica, Chipre, Chequia, Dinamarca, Estonia, Finlandia, Francia, Alemania, Grecia, Hungría, Irlanda, Italia, Lavinia, Lituania, Luxemburgo, Malta, Holanda, Noruega, Polonia, Portugal, Eslovaquia, Eslovenia, España, Suecia, Suiza y Reino Unido, con sede en Bruselas.

2 Centro de Investigación sobre la Sociedad del Conocimiento (CIC), vinculado al Instituto Universitario de Administración de Empresas de la Universidad Autónoma de Madrid.

3 Desde que en 1994 Thomas Stewart publicó en la revista Fortune su artículo “Intelectual Capital: Your Company's Most Valuable Asset", han proliferado un gran numero de expresiones para hacer referencia a la riqueza inmaterial de las organizaciones. El modelo Intellectus ha optado por establecer una coherente diferenciación de todos estos términos con el fin de distinguir la pluralidad de matices característica de esta área de investigación.

4 Con base en que, por ejemplo, un programador de Java en India cuesta 5.000 dólares anuales frente a los 60.000 dólares de Estados Unidos, además de que estos trabajadores cuentan con amplios conocimientos de inglés por lo que el idioma no es un problema.

5 Desde 1974 hasta el final de la Ronda Uruguay, el comercio de textiles se rigió por el Acuerdo Multifibras (AMF), que sirvió de marco a acuerdos bilaterales o medidas unilaterales de establecimiento de contingentes, por lo que se limitaban las importaciones de países en los que el rápido aumento de esas importaciones representaba un grave perjuicio para las ramas de producción nacionales.A partir de 1995, el Acuerdo Multifibras quedó sustituido por el Acuerdo sobre los Textiles y el Vestido (ATV), de la OMC. Para el $1^{\text {o }}$ de enero de 2005 el sector estaba de estar plenamente integrado en las disposiciones normales del GATT.

6 Central American Free Trade Agreement (CAFTA), firmado en mayo de 2004 entre Estados Unidos, Guatemala, El Salvador, Honduras, Costa Rica, y Nicaragua.

\section{BIBLIOGRAFIA}

Aktouf, Omar. (2004). Pós-globalização, administração e racionalidade econômica: a Síndrome do Avestruz. São Paulo, ATLAS.

Badaracco Jr., Joseph L. (1992). Alianzas Estratégicas: El caso de General Motors e IBM. Madrid, McGraw-Hill.

Bender, Silke; y Fish, Alan. (2000). The transfer of knowledge and the retention of expertise: the continuing need for global assignments. Journal of Knowledge Management, vol. 4, № 2, pp. 125-137.

Bontis, N. (1998). Intellectual Capital: an exploratory study that develops measures and models. Management Decision, vol. 36, № 2, pp. 67-76.

Bordo, Michael D. (2002). Globalization in historical perspective. Business Economics, January 2002.

Brooking, A. (1996). Intellectual Capital: Core asset for the third millennium enterprise. London: International Thomson Business Press.

Bueno, E. (2002). Dirección estratégica basada en conocimiento: Teoría y práctica de la nueva perspectiva. En P. Morcillo y J. Fernández Aguado (Eds.): Nuevas claves en la dirección estratégica. Madrid, Ariel, pp. 91-116.

Camisón, C.; Palacios, D.; Devece, C. (2000). Un nuevo modelo para la medición del capital intelectual: el modelo Nova. Ponencia presentada al X Congreso de ACEDE, Oviedo.

Ceballos Fernández, Pedro; Colmena, Pedro M. (2004): La deslocalización. Presentación disponible en $w w w$.deslocalizacion.tk, acceso el 09 de diciembre del 2004. 
Comité Européen de Normalisation (CEN) (2004). European Guide to good Practice in Knowledge Management. Brussels: Workshop Agreement, March 2004.

Centro de Investigación sobre la Sociedad del Conocimiento (CIC). Instituto Universitario de Administración de Empresas - Universidad Autónoma de Madrid. (2003). Modelo Intellectus: Medición y Gestión del Capital Intelectual. Madrid, CIADE, UAM.

Drucker, Peter F. (1999). Desafios gerenciais para o século XXI. São Paulo, Pioneira.

Edvinsson, Leif; Malone, Michael S. (1999). El Capital Intelectual: cómo identificar y calcular el valor de los recursos intangibles de su empresa. Barcelona, Gestión 2000.

Fernández, Carlos M. y Myro, Rafael. (1996). The migrations of the industrial activity inside and outside the European Union. ETH Zurich, Switzerland; European Regional Science Association, $36^{\text {th }}$ European Congress.

Guarasa, Marta M. (2002). Deslocalización sectorial de la inversión directa extranjera en España. Boletín Económico de ICE - Información Comercial Española, № 2744, pp. $19-30$.

Hofstede, Geert. (1991). Cultures and Organizations: Software of the Mind. London, McGraw-Hill.

Kaplan, R.S.; y Norton, D.P. (1996). The Balanced Scorecard. Massachussets, Harvard Business School Press.

Nonaka, I.; Takeuchi, H. (1995). The Knowledgecreating Company: How Japanese Companies Create the Dynamics of
Innovation. New York, Oxford University Press.

Nonaka, I.; Takeuchi, H. (1997). Criação de conhecimento na empresa. Rio de Janeiro, Campus.

Prusak, L. (1996). The Knowledge Advantage. Planning Review, vol. 24, № 2, pp. 6-8.

Rodríguez, M.F.G. (2004). La internacionalización de las empresas españolas. Revista Historia Empresarial № 812, enero.

Saint Onge, H. (1996). Tacit Knowledge: the key to the strategic alignment of intellectual capital. Strategy and Leadership, vol. 24, № 2 , pp. 10-14.

Sanguino, Ramón. (2005). Gestión del Conocimiento y Competitividad: Análisis en las Ciudades Españolas. Tesis Doctoral. Badajoz - España, Universidad de Extremadura.

Stewart, T. A. (1999). Intellectual Capital: The new wealth of organizations. London, Nicholas Brealey Publishing.

Sveiby, Karl Erik. (1998). A nova riqueza das organizações: gerenciando e avaliando patrimônios de conhecimento. Rio de Janeiro, Campus.

Sveiby, Karl Erik. (2000). O valor do intangível. HSM Management 22 Año 4, Setembro Outubro.

Vera, D.; y Crossan, M. (2004). Strategic leadership and organizational learning. Academy of Management Review, v. 29, № 2, pp. 222240.

Zabot, João B.M. (2002). Gestão do Conhecimento: Aprendizagem e Tecnologia Construindo a Inteligência Coletiva. São Paulo, ATLAS. 